\title{
液相辅助热压烧结制备 $\mathrm{Cu}(\mathrm{In}, \mathrm{Ga}) \mathrm{Se}_{2}$ 靶材的研究
}

\author{
黄耀芹 ${ }^{1}$, 郑国源 ${ }^{1}$, 莫淑一 ${ }^{1}$, 何丽秋 ${ }^{2}$, 王东生 ${ }^{2}$, 龙飞 ${ }^{1}$
}

(1. 桂林理工大学 材料科学与工程学院, 广西高校有色金属清洁冶炼与综合利用重点实验室, 桂林 541004; 2. 广 西地凯光伏能源有限公司, 南宁 530003)

摘 要: 采用 $\mathrm{CuIn}_{0.7} \mathrm{Ga}_{0.3} \mathrm{Se}_{2} 、 \mathrm{In}_{0.7} \mathrm{Ga}_{0.3} \mathrm{Se}$ 和 CuSe 为原料, 一步热压烧结法制备 CIGS 靶材。通过阿基米德法、 XRD、Raman、SEM 和 EDS 等测试方法研究了 CuSe 液相辅助烧结对靶材的相对密度、物相组成、结构、形貌以 及成份的影响。结果表明, 加入 CuSe 在高温下产生液相能显著促进烧结, 比不加入 CuSe 的体系的烧结温度下降 了 $50^{\circ} \mathrm{C}$ 。当烧结温度为 $575^{\circ} \mathrm{C}$ 时, 获得的靶材相对密度为 $96.18 \%$, 物相单一纯净, 微观结构致密, 晶粒发育良好。 以摩尔比为 $\mathrm{Cu}: \mathrm{In}: \mathrm{Ga}: \mathrm{Se}=23.0: 18.2: 6.5: 52.3$ 的靶材, 通过溅射-热处理法制备 CIGS 薄膜, 并完成 $\mathrm{AZO} / \mathrm{ZnO} /$ $\mathrm{CdS} / \mathrm{CIGS} / \mathrm{Mo}$ 单电池的组装 $\left(0.25 \mathrm{~cm}^{2}\right)$, 获得了 $9.6 \%$ 的光电转换效率。

关 键 词: CIGS; 热压烧结; 靶材; 转换效率

中图分类号: TN304 文献标识码: A

\section{Liquid-assisted Hot-pressed Sintering of $\mathrm{Cu}(\mathrm{In}, \mathrm{Ga}) \mathrm{Se}_{2}$ Targets}

\author{
HUANG Yao-Qin ${ }^{1}$, ZHENG Guo-Yuan ${ }^{1}$, MO Shu-Yi ${ }^{1}$, HE Li-Qiu ${ }^{2}$, WANG Dong-Sheng ${ }^{2}$, LONG Fei $^{1}$ \\ (1. Guangxi Key Laboratory in Universities of Clean Metallurgy and Comprehensive Utilization for Non-ferrous Metals Re- \\ sources, College of Materials Science and Engineering, Guilin University of Technology, Guilin 541004, China; 2. Guangxi \\ Dikai PV Energy Co., Ltd, Nanning 530003, China)
}

\begin{abstract}
CIGS target was prepared by one step hot-pressing sintering using $\mathrm{CuIn}_{0.7} \mathrm{Ga}_{0.3} \mathrm{Se}_{2}, \mathrm{In}_{0.7} \mathrm{Ga}_{0.3} \mathrm{Se}$ and CuSe as precursor powder. Archimedes method, XRD, Raman, SEM, and EDS were carried out to investigate the effects of liquid-assisted sintering on the relative density, phase, structure, morphology, and composition of CIGS target. The results showed that CuSe would turn into liquid phase when temperature was increased to the liquid phase points. The liquid phase can significantly reduce sintering temperature (about $50{ }^{\circ} \mathrm{C}$ dropping) and accelerate the sintering process. When sintered at $575^{\circ} \mathrm{C}$, the target can obtain the relative density of $96.18 \%$, single phase, dense microstructure with good grain growth. In addition, using the target with molar ratio of $\mathrm{Cu}: \mathrm{In}: \mathrm{Ga}: \mathrm{Se}=23.0: 18.2: 6.5: 52.3$, CIGS thin films were also prepared by sputtering-heat treatment, followed assembled into AZO/ZnO/CdS/CIGS/Mo solar cell. The fabricated CIGS solar cells presented a photoelectric conversion efficiency of $9.6 \%$.
\end{abstract}

Key words: CIGS; hot-pressing sintering; target; conversion efficiency

铜铟镓硒(CIGS) 薄膜太阳电池具有抗辐射能力 强、工作性能稳定和光电转换效率高等优点, 应用 前景广阔。CIGS 太阳电池吸收层的制备方法主要
有共蒸发法 ${ }^{[1-2]}$ 、磁控溅射-硒化法 ${ }^{[3-6]}$ 和单靶溅射一 步法 ${ }^{[7-8]}$ 等。虽然共蒸发法获得的薄膜质量较好, 但 是生产工艺复杂、原料利用率较低、样品成品率不

收稿日期: 2016-03-28; 收到修改稿日期：2016-05-02

基金项目: 广西自然科学基金(2014GXNSFFA118004); 教育部 “新世纪优秀人才支持计划” (NCET-12-0655) Guangxi Natural Science Foundation (2014GXNSFFA118004); The Program for New Century Excellent Talents in University (NCET-12-0655)

作者简介：黄耀芹(1991-), 女, 硕士研究生. E-mail: m15296818093@163.com

通讯作者: 龙飞，教授. E-mail: longf@glut.edu.cn 
高, 大面积制备的工艺要求非常苛刻。磁控溅射-硒化 法容易进行大面积制备, 但是后期硒化工艺复杂、工 艺时间长、设备要求高, 并有潜在的安全问题和环境 负担。单靶一步溅射法在溅射一硒化法基础上省去后 期硒化工艺，提高了生产效率，有利于制备大面积 CIGS 薄膜。而采用该方法制备高质量 CIGS 薄膜的关 键在于高密实率相对密度的 CIGS 陶瓷靶材。

Suryanarayana 等 ${ }^{[9]}$ 以铜、铟、镓、硒单质粉为 原料, 利用机械合金化制备 CIGS 四元粉, 采用热等 静压制备了 CIGS 靶材; Tamura 等 ${ }^{[10]}$ 采用类似方法 获得了相对密度达到 98.9\%的靶材。但该方法制备 成本过高, 不适用商业化生产。Hsu 等 ${ }^{[11]}$ 使用 CIGS 纳米粉, 在 $550^{\circ} \mathrm{C}$ 保温 $3 \mathrm{~h}$ 获得了相对密度为 $92 \%$ 的 CIGS 靶材。王东生等 ${ }^{[12]}$ 采用固相反应法制备出 CIGS 前驱粉, 并将粉末冷等静压后, 高温烧结得到 CIGS 靶材。2010 年, 庄大明等 ${ }^{[13]}$ 利用 $\mathrm{Cu}_{2} \mathrm{Se}+$ $\mathrm{In}_{2} \mathrm{Se}_{3}+\mathrm{Ga}_{2} \mathrm{Se}_{3}$ 混合粉末经过 $1000 \mathrm{MPa}$ 的冷等静压 之后, 在 $\mathrm{H}_{2}$ 气氛中 $850^{\circ} \mathrm{C}$ 无压烧结 $2 \mathrm{~h}$ 得到相对密 度为 $89 \%$ 的 CIGS 靶材。冷等静压-无压烧结工艺烧 结温度高, 容易造成组份挥发。此后, 该课题组系统 研究了热压烧结工艺对 CIGS 靶材的影响 ${ }^{[14]}$, 采用(In, $\mathrm{Ga})_{2} \mathrm{Se}_{3}$ 和 $\mathrm{Cu}_{2} \mathrm{Se}$ 为原料, 在 $45 \mathrm{MPa}$ 压力下 $900^{\circ} \mathrm{C}$ 保 温 $4 \mathrm{~h}$ 获得了相对密度为 $96.82 \%$ 的靶材。

根据 $\mathrm{Cu}-\mathrm{Se}$ 二元相图, $\mathrm{Cu}: \mathrm{Se}=1: 1$ 的硒化铜熔 点最低, 为 $523^{\circ} \mathrm{C}^{[15]}$ 。本工作即以 $\mathrm{CuSe}$ 作为低温液 相源，通过对比 $\mathrm{CuIn}_{0.7} \mathrm{Ga}_{0.3} \mathrm{Se}_{2}+\mathrm{In}_{0.7} \mathrm{Ga}_{0.3} \mathrm{Se}+\mathrm{CuSe}$ 配方和纯 $\mathrm{CuIn}_{0.7} \mathrm{Ga}_{0.3} \mathrm{Se}_{2}$ 配方的烧结行为, 考察有/ 无液相辅助热压烧结对靶材物相组成、形貌、成份 和烧失量的影响。通过研究 CuSe 的引入对 CIGS 靶材烧结性能的影响, 探索在较低烧结温度下热压 烧结制备高密实度靶材的工艺可行性。并在实验结 果的基础上, 分析液相辅助热压烧结制备 CIGS 靶 材的过程。最后采用制备的高质量靶材一步溅射制 备 CIGS 薄膜, 组装成太阳能电池器件, 采用电池转 换效率测试仪测定 CIGS 电池器件的光电转换效率。

\section{1 实验方法}

\subsection{CIGS 靶材的制备}

实验原料为自制的 $\mathrm{CuIn}_{0.7} \mathrm{Ga}_{0.3} \mathrm{Se}_{2} 、 \mathrm{In}_{0.7} \mathrm{Ga}_{0.3} \mathrm{Se}$ 和 CuSe 粉体, CuIn ${ }_{0.7} \mathrm{Ga}_{0.3} \mathrm{Se}_{2}$ 粒径为 $500 \sim 1000 \mathrm{~nm}$, CuSe 和 $\mathrm{In}_{0.7} \mathrm{Ga}_{0.3}$ Se 粒径为 $100 \sim 200 \mathrm{~nm}$ 。其中, A 配方原料粉为 $\mathrm{CuIn}_{0.7} \mathrm{Ga}_{0.3} \mathrm{Se}_{2}$ 四元粉; $\mathrm{B}$ 配方原料 粉为混合均匀的 $\mathrm{CuIn}_{0.7} \mathrm{Ga}_{0.3} \mathrm{Se}_{2} 、 \mathrm{In}_{0.7} \mathrm{Ga}_{0.3} \mathrm{Se}$ 和 CuSe, 三者摩尔比为 $1: 0.5: 0.5$, 混料以无水乙
醇为球磨介质，经行星球磨 $(280 \mathrm{r} / \mathrm{min})$ 混料 $12 \mathrm{~h}$ 后 在真空干燥箱中 $80^{\circ} \mathrm{C}$ 真空干燥 $12 \mathrm{~h}$, 干燥后的粉 末经 $74 \mu \mathrm{m}$ 笁网过篮即可。采用热压烧结工艺制备 CIGS 靶材, 氞气作为保护气氛, 烧结温度为 $525 \sim 625^{\circ} \mathrm{C}$, 升温速率为 $10^{\circ} \mathrm{C} / \mathrm{min}$, 压力为 $40 \mathrm{MPa}$, 保温时间为 $2 \mathrm{~h}$ 。

\subsection{CIGS 薄膜太阳电池器件组装}

以钠钙玻璃为基板, 清洁后采用磁控溅射工艺 在其上制备 Mo 层作为背电极。以前述热压烧结制 备的 CIGS 四元靶为原料, 采用单靶一步溅射法制 备 CIGS 薄膜。采用水浴法制备 CdS, 射频浌射法 制备 $\mathrm{ZnO}$, 直流溅射制备 $\mathrm{AZO}$ 透明上电极, 完成 $\mathrm{AZO} / \mathrm{ZnO} / \mathrm{CdS} / \mathrm{CIGS} / \mathrm{Mo}$ 单电池的组装。

\section{3 表征方法}

采用 X-Pert PRO MRD 型 X 射线衍射仪对样品 进行物相分析; 采用美国热电公司生产的 Thermo Fisher Scientific DXR 激光共聚焦显微拉曼光谱仪 对样品的物质组分进行分析; 采用日本日立公司 生产的 S-4800 型场发射扫描电子显微镜(FESEM) 对制备的靶材和薄膜微观形貌、晶粒大小及其分布 情况进行表征; 采用由场发射扫描电镜自带的 INCA IE 350 型能谱分析仪(EDS)进行成份测定; 采用阿基米德排水法测试 CIGS 靶材的密度, 并与 $\mathrm{CuIn}_{0.7} \mathrm{Ga}_{0.3} \mathrm{Se}_{2}$ 理论密度值 $5.69 \mathrm{~g} / \mathrm{cm}^{3}$ 相比计算其 相对密度; 采用光伏组件测试仪(型号: GSMT-H-A) 测定 CIGS 电池器件在仿太阳光全光谱金属卤素灯 (光源功率为 $100 \mathrm{~mW} / \mathrm{cm}^{2}$ )下的转换效率。

\section{2 结果与讨论}

\section{1 液相辅助对 CIGS 靶材物相的影响}

A、B 配方分别在不同温度烧结得到的 CIGS 靶材的 XRD 图谱(图 1)与标准 PDF 卡(JCPDS 35-1102)一致, 均为单一的四元相 $\mathrm{CuIn}_{0.7} \mathrm{Ga}_{0.3} \mathrm{Se}_{2}$ 。 $2 \theta=26.897^{\circ} 、 44.645^{\circ} 、 52.944^{\circ}$ 出现的衍射峰, 分别 对应 CIGS 标准 PDF 卡(JCPDS 35-1102)中的(112)、 (220)、(312)晶面。相比之下, B 配方获得的靶材衍射 峰强度比配方 $\mathrm{A}$ 的更高, 这是由于配方 $\mathrm{B}$ 中添加了 CuSe, 而 CuSe 在烧结温度范围会产生液相(CuSe 的 熔点为 $523^{\circ} \mathrm{C}$ ), 促进了烧结。另外, B 配方在 $525^{\circ} \mathrm{C}$ 温 度下保温 $2 \mathrm{~h}$, 所得样品的物相中没有出现 CuSe 和 $\mathrm{In}_{0.7} \mathrm{Ga}_{0.3} \mathrm{Se}$ 的衍射峰，表明在该温度下保温一段时间 二者已经发生反应生成了四元相 $\mathrm{CuIn}_{0.7} \mathrm{Ga}_{0.3} \mathrm{Se}_{2}$ 。

A、B 配方烧结得到的靶材的半高宽值都随着 烧结温度的升高而降低(图 2), 说明在烧结过程中, 


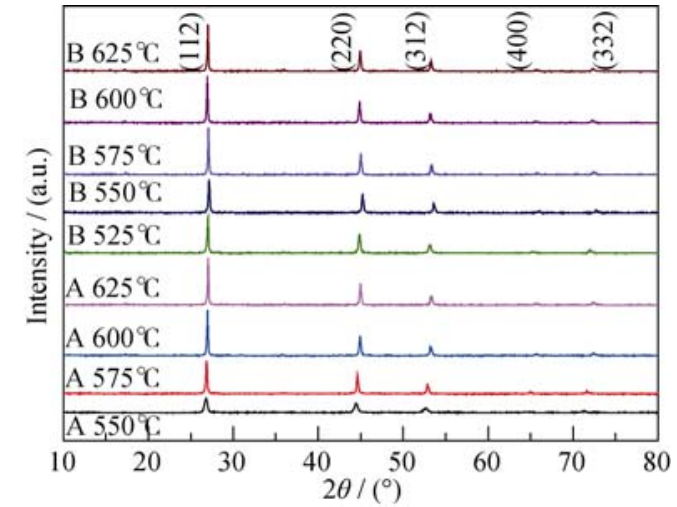

图 1 A、B 配方在不同温度烧结所得靶材的 XRD 图谱 Fig. 1 XRD patterns of targets sintered at different temperatures from formula A and B powders

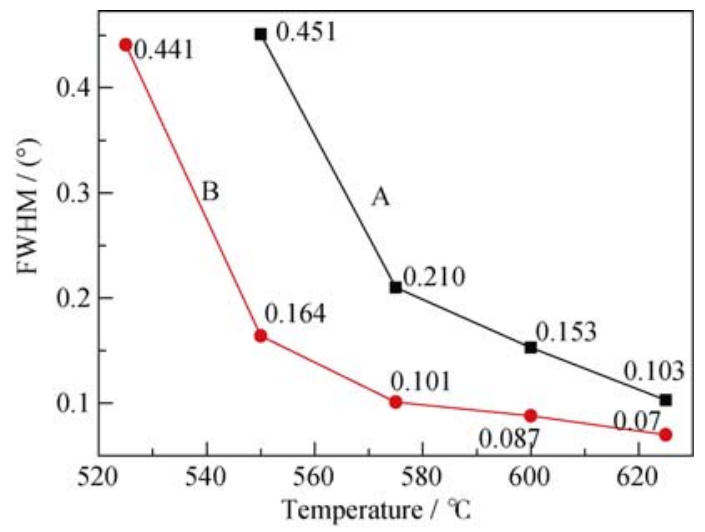

图 2 A、B 配方在不同烧结温度所得靶材的 XRD 衍射峰第 一强峰的半高宽

Fig. 2 FWHM of main diffraction peak of targets sintered at different temperatures from formula A and B powders

晶粒不断长大, 结晶程度不断增大。相比之下, $\mathrm{B}$ 配 方在 $575^{\circ} \mathrm{C}$ 烧结得到靶材的半高宽值略小于 A 配方 在 $625^{\circ} \mathrm{C}$ 烧结得到靶材的半高宽值, 这表明在添加 CuSe 的配方中, CuSe 高温下产生液相, 促进传质过 程以及晶粒的生长, 提高了靶材的结晶程度, 从而 降低了烧结温度。

\section{2 拉曼图谱分析}

靶材的 Raman 图谱显示, $162.1 \mathrm{~cm}^{-1}$ 为 CIGS 黄 铜矿的 A1 特征峰, $202.6 \mathrm{~cm}^{-1}$ 为 CIGS 黄铜矿的 B2 或 $\mathrm{E}$ 的特征峰, 与文献[16]报道值相符。结合 XRD 图 谱可知靶材的物相单一纯净, 不存在二次相(图 3)。

\section{3 液相辅助烧结对 CIGS 靶材相对密度的 影响}

A、B 配方原料混合粉不经预压成型，直接装到 石墨模具后装炉, 炉内充入高纯氩气作为保护气氛, 以 $10^{\circ} \mathrm{C} / \mathrm{min}$ 的升温速度升到额定温度, 加压(压力 为 $40 \mathrm{MPa}$ )并保温 $2 \mathrm{~h}$ 。不同温度下热压烧结制得的 靶材相对密度情况如图 4 所示。

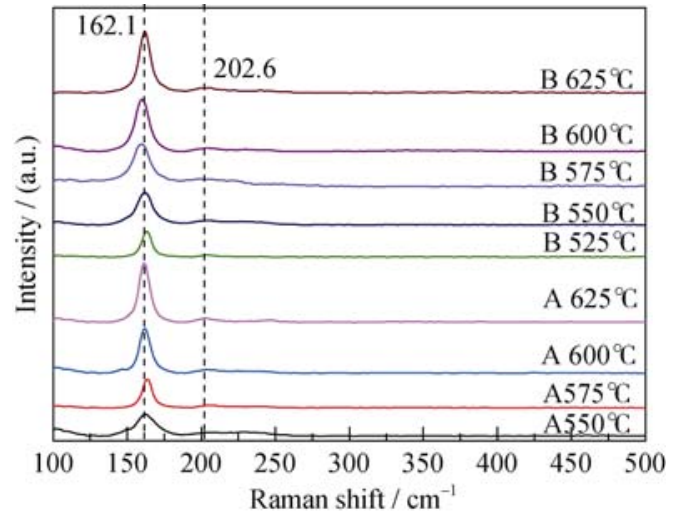

图 3 A、B 配方在不同温度烧结所得靶材的 Raman 图谱

Fig. 3 Raman spectra of the targets sintered at different temperatures from formula A and B powders

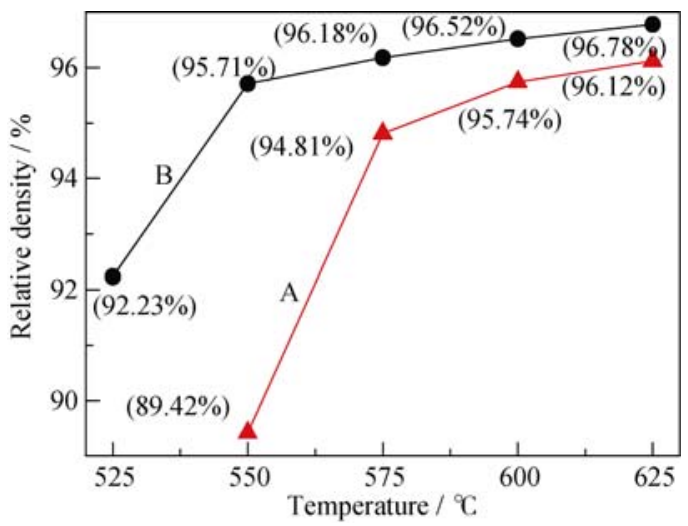

图 4 不同烧结温度所得靶材的相对密度

Fig. 4 Relative density of targets sintered at different temperatures

当烧结温度从 $550^{\circ} \mathrm{C}$ 升高至 $575^{\circ} \mathrm{C}$ 时, $\mathrm{A}$ 配方靶 材的相对密度从 $89.42 \%$ 提高至 $94.81 \%$, 致密度提高 了 $5.45 \%$; 当烧结温度从 $575^{\circ} \mathrm{C}$ 提高至 $625^{\circ} \mathrm{C}$, A 配方 靶材的相对密度从 94.81\%提高至 96.12\%, 致密度仅 提高了 $1.31 \%$ 。由此可推断靶材在 $550 \sim 575^{\circ} \mathrm{C}$ 的温度 区间扩散速率剧增, 靶材体积收缩最大。而当烧结温 度为 $525^{\circ} \mathrm{C}$ 时, B 配方靶材的相对密度为 $92.23 \%$; 在烧 结温度为 $550 \sim 625^{\circ} \mathrm{C}$ 区间内, 靶材相对密度曲线平缓, 这是因为该配方在烧结温度为 $550^{\circ} \mathrm{C}$ 时, 靶材的相对 密度已高达 $95.71 \%$, 即使继续提高烧结温度至 $625^{\circ} \mathrm{C}$, 靶材的致密度增幅很小。实验结果表明当烧结温度为 $575^{\circ} \mathrm{C}$ 时, 整个烧结体系已趋于平衡, 再继续升高温 度, 对靶材的烧结致密度影响不大。

值得注意的是，烧结温度为 $550^{\circ} \mathrm{C}$ 时, $\mathrm{A}$ 配方靶 材的相对密度仅为 $89.42 \%$, B 配方获得靶材的相对 密度则高达 $95.71 \%$ 。B 配方在 $575^{\circ} \mathrm{C}$ 烧结获得的靶 材的相对密度高于 A 配方在 $625^{\circ} \mathrm{C}$ 烧结获得的靶材。 这是由于 CuSe 液相点在 $523^{\circ} \mathrm{C}^{[15]}$, B 配方体系中由于 存在 CuSe, 在较低温度下即可产生液相, 产生的该液 相一方面促进原料的传质, 提高烧结速率; 另一方面, 
在液相的毛细管力作用下, 颗粒发生重排, 气孔随着 物质的迁移迅速排出体外, 得到相对密度高的靶材 ${ }^{[13]}$ 。 同时, 处于 $\mathrm{CuIn}_{0.7} \mathrm{Ga}_{0.3} \mathrm{Se}_{2}$ 晶粒空隙处的 CuSe 液相在 高温下与 $\mathrm{In}_{0.7} \mathrm{Ga}_{0.3} \mathrm{Se}$ 反应生成 $\mathrm{CuIn}_{0.7} \mathrm{Ga}_{0.3} \mathrm{Se}_{2}$ 半空隙填 实, 进一步提高了烧结致密度。

通过以上分析可以推断, 添加 CuSe 辅助剂有效降 低了 CIGS 靶材的烧结温度, 提高了靶材的相对密度。

\section{4 液相辅助烧结对 CIGS 靶材微观结构的 影响}

A 配方在 $600^{\circ} \mathrm{C}$ 基本烧结, 获得的靶材内部气 孔不多, 晶粒相互连接紧密, 还没有发育出明显的 晶界(图 5(a))。当烧结温度提高至 $625^{\circ} \mathrm{C}$ 时, 靶材内 部晶粒自形程度不高, 晶界仍不明显, 但是靶材内 部气孔有所减少(图 5(b))。B 配方在 $550^{\circ} \mathrm{C}$ 时即实现了 烧结, 靶材内部的气孔率极低, 晶粒轮廓开始出现(图 $5(c))$ 。当烧结温度提高至 $575^{\circ} \mathrm{C}$, 由于升温速度较快, 当样品快速升温并超过 CuSe 熔点后, CuSe 的粘度迅 速下降, 传质速度得到显著的提升; 同时, 液相的出 现也会加快周围的 $\mathrm{CuIn}_{0.7} \mathrm{Ga}_{0.3} \mathrm{Se}_{2}$ 晶粒的生长速度, 由于传质过程相对较充分, 晶体发育较完整(图 5(d))。

对比发现, B 配方 $550^{\circ} \mathrm{C}$ 烧结获得的靶材显微结 构与 $\mathrm{A}$ 配方在 $600^{\circ} \mathrm{C}$ 烧结获得的靶材较为接近; 而 $\mathrm{B}$ 配方在 $575^{\circ} \mathrm{C}$ 烧结所得靶材(图 5(d))致密度和结晶度 也略好于 A 配方在 $625^{\circ} \mathrm{C}$ 烧结获得的靶材(图 5(b)), 这 说明加入 CuSe 使体系的烧结温度至少下降了 $50^{\circ} \mathrm{C}$ 。

液相辅助烧结行为过程示意图如图 6 所示。 CuSe 高温下形成液相并对烧结产生显著的促进作 用，其机理总结如下：一方面液相促进了原料的传 质, 粉体颗粒在液相里呈近似悬浮状态, 受液相表
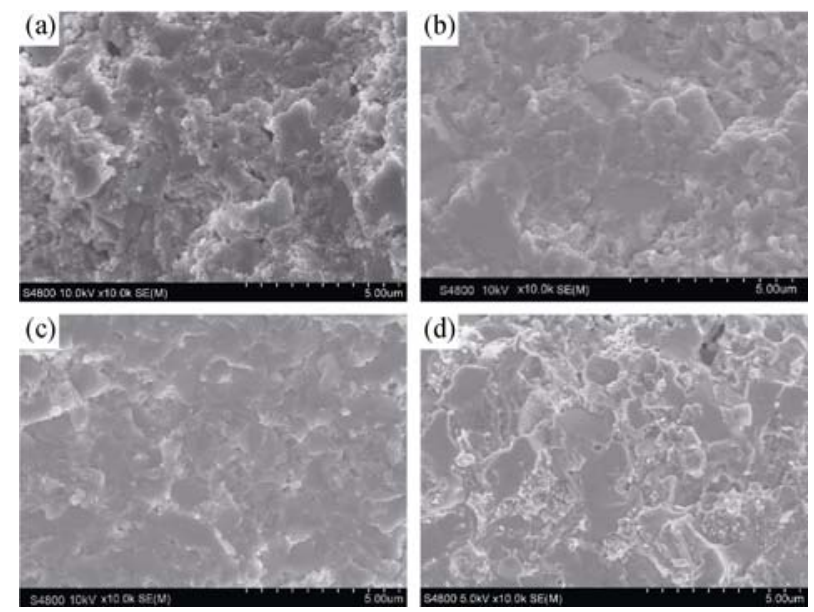

图 5 不同配方在不同温度烧结所得 CIGS 靶材的断面形貌 FESEM 照片

Fig. 5 Cross-sectional FESEM images of CIGS targets sintered at different temperatures from different formula powders

(a) Formula A, $T=600^{\circ} \mathrm{C}$; (b) Formula A, $T=625^{\circ} \mathrm{C}$; (c) Formula B, $T=550^{\circ} \mathrm{C}$; (d) Formula B, $T=575^{\circ} \mathrm{C}$

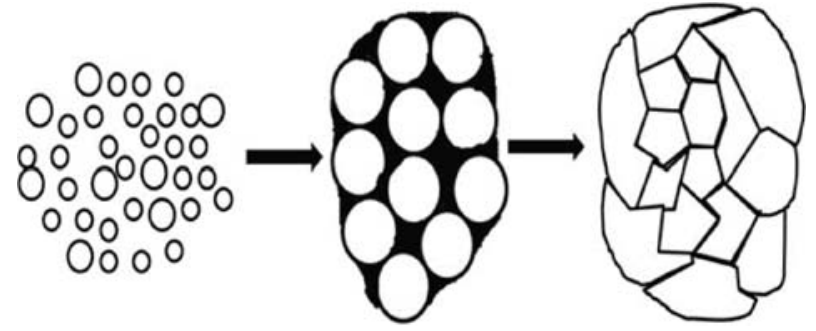

图 6 液相辅助烧结晶粒生长发育原理示意图

Fig. 6 Principle diagram of the crystal growth in liquid-assisted sintering

面张力的推动发生扩散, 晶粒快速长大, 提高了烧 结速率; 另一方面，在液相的毛细管力作用下，颗 粒间产生压力并使其易于滑动, 导致颗粒重排并改 善颗粒的堆积结构。颗粒之间有液相存在时, 颗粒 互相压紧, 又提高了固体物质在液相中的溶解度, 晶粒发生重组, 这样可以促进气体的排出, 流动传 质使烧结致密化速率比纯固相烧结有了很大的提 高 ${ }^{[13,17-19]}$ 。并且, $\mathrm{CuIn}_{0.7} \mathrm{Ga}_{0.3} \mathrm{Se}_{2}$ 颗粒在烧结过程中 起晶种的作用, 处于 $\mathrm{CuIn}_{0.7} \mathrm{Ga}_{0.3} \mathrm{Se}_{2}$ 晶粒空隙处的 CuSe 液相与 $\mathrm{In}_{0.7} \mathrm{Ga}_{0.3} \mathrm{Se}$ 反应生成 $\mathrm{CuIn}_{0.7} \mathrm{Ga}_{0.3} \mathrm{Se}_{2}$ 半 空隙填实, 进一步提高了靶材密实度。

实验对升温速率也进行了简单研究, 发现升温 速度过慢, CuSe 还没熔解, 便直接与 $\mathrm{In}_{0.7} \mathrm{Ga}_{0.3} \mathrm{Se}$ 发 生反应完全生成 $\mathrm{CuIn}_{0.7} \mathrm{Ga}_{0.3} \mathrm{Se}_{2}$, 整个烧结过程无 法产生 CuSe 液相。升温速率快, 可以使得烧结温度 快速升至 CuSe 的熔点, CuSe 熔解形成液相, 再与 $\mathrm{In}_{0.7} \mathrm{Ga}_{0.3} \mathrm{Se}$ 发生反应, 液相的产生以及化学反应推 动力, 使得靶材相比慢速升温更易烧结, 从而获得 高致密度的靶材。

\section{5 液相辅助对 CIGS 靶材成份与烧失量的 影响}

表 1 中 a、b、c、d 对应 A 配方; e、f、g、h、i 对应 B 配方。两种配方的靶材化学计量比均接近理 论的原子百分比 $1: 0.7: 0.3: 2$ 。无论是 $A$ 配方还 是 B 配方, 靶材的烧失量都会随着烧结温度的升高 而上升。相比之下, B 配方的烧失量都低于 A 配方。 由此表明, 添加 CuSe 作辅助烧结剂, 烧结过程中液 相的产生可以有效促进靶材在较低温度快速烧结 (与前述分析相一致), 抑制 $\mathrm{CuIn}_{0.7} \mathrm{Ga}_{0.3} \mathrm{Se}_{2}$ 的分相和 挥发, 从而保持了靶材成份比例和烧失量的稳定性, 与 XRD 分析结果相吻合。

\subsection{CIGS 薄膜结构性能分析}

采用 $\mathrm{B}$ 配方在 $575^{\circ} \mathrm{C}$ 烧结得到的靶材溅射制备 CIGS 薄膜, 组装成电池, 根据电池转换效率的结果对 靶材成份进行了调整。最后采用摩尔比为 $\mathrm{Cu}: \mathrm{In}$ ： 
Ga $:$ Se $=23.0: 18.2: 6.5: 52.3$ 的靶材, 在镀钿的玻 璃基板上射频溅射制备 CIGS 薄膜, 该薄膜经氩气气
氛下 $550^{\circ} \mathrm{C}$ 热处理 $30 \mathrm{~min}$, 获得的薄膜物相单一纯净 (图 7(a, b)), 表面平整, 晶粒尺寸约为 $0.5 \mu \mathrm{m}$ (图 7(c)),

表 1 不同配方所得靶材的成份比例与烧失量

Table 1 Composition proportion and loss on ignition table of targets by different formula

\begin{tabular}{cccccccc}
\hline Sample & Additive CuSe & Temperature $/{ }^{\circ} \mathrm{C}$ & $\mathrm{Cu} /$ at\% & In / at\% & Ga /at\% & Se / at\% & Loss on ignition / \% \\
\hline Raw material & No & -- & 24.98 & 17.52 & 7.49 & 50.01 & -- \\
a & No & 550 & 25.18 & 17.44 & 7.49 & 49.89 & 3.11 \\
b & No & 575 & 24.96 & 17.41 & 7.56 & 50.07 & 3.44 \\
c & No & 600 & 25.22 & 17.43 & 7.39 & 49.96 & 3.75 \\
d & No & 625 & 25.19 & 17.49 & 7.59 & 49.73 & 3.88 \\
\hline Raw material & Yes & -- & 25.02 & 17.49 & 7.47 & 50.02 & -- \\
e & Yes & 525 & 24.98 & 17.46 & 7.48 & 50.08 & 0.49 \\
f & Yes & 550 & 24.89 & 17.42 & 7.45 & 50.24 & 0.59 \\
g & Yes & 575 & 25.04 & 17.52 & 7.55 & 49.89 & 0.67 \\
h & Yes & 600 & 25.12 & 17.48 & 7.52 & 49.88 & 0.74 \\
i & Yes & 625 & 24.78 & 17.38 & 7.45 & 50.39 & 0.83 \\
\hline
\end{tabular}
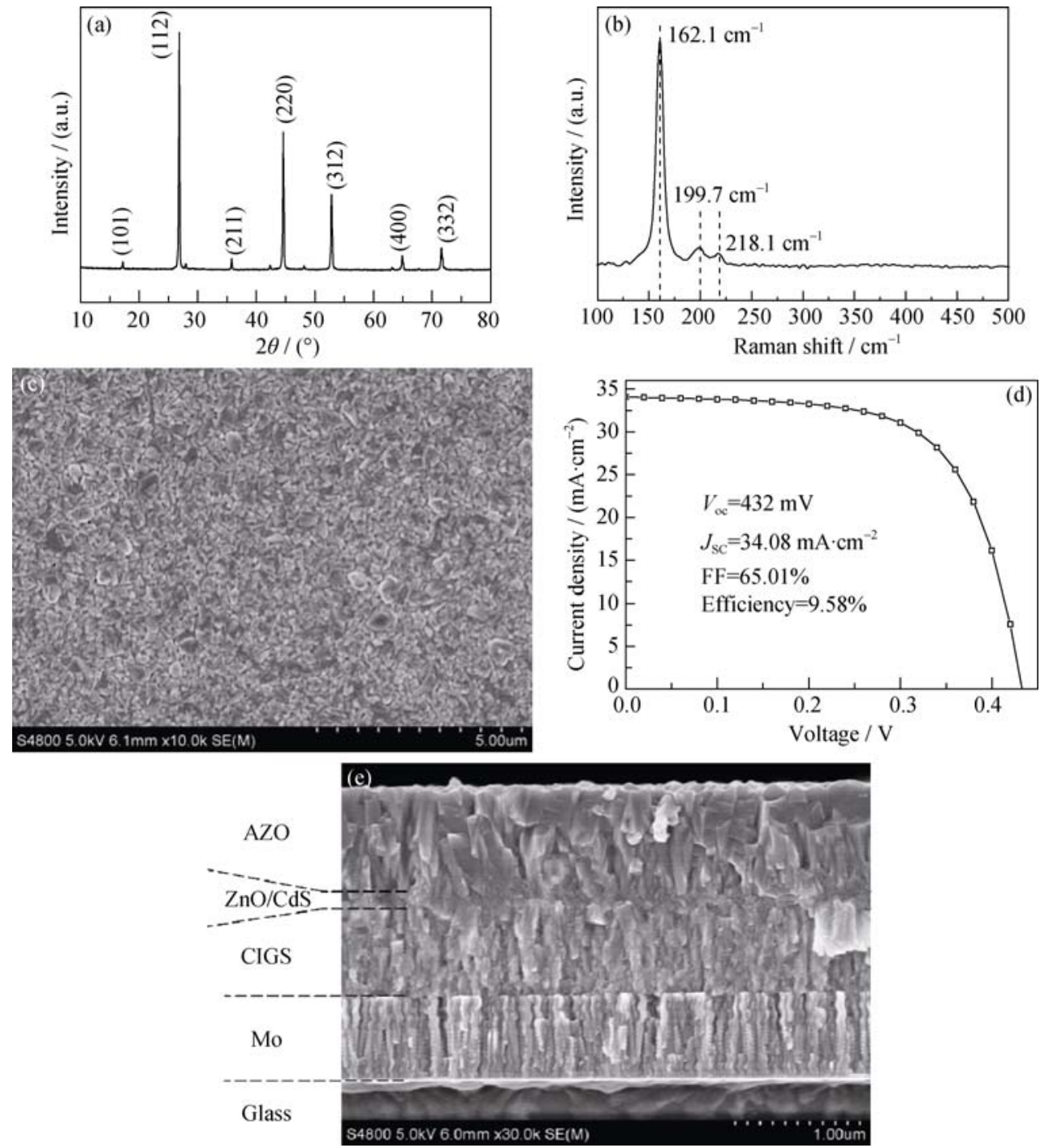

图 7 溅射-热处理制备的 CIGS 薄膜 XRD 图(a), 拉曼光谱图(b)和表面 FESEM 照片(c), 单电池的 $I-V$ 曲线图(d), 以及截面的 FESEM 照片(e)

Fig. 7 XRD pattern(a), Raman pattern(b) and plan view (c) of CIGS thin film preparation by sputtering-heat treatment, and its $I$ - $V$ pattern(d) and cross-sectional view (e) FESEM image of a single battery 
膜厚约为 $800 \mathrm{~nm}$, 按电池器件组装工艺得到的 $\mathrm{AZO} / \mathrm{ZnO} / \mathrm{CdS} / \mathrm{CIGS} / \mathrm{Mo}$ 单电池截面结构清晰(图 7(e)), 电池转换效率为 9.6\%(图 7(d))。

本实验中，较佳的 CIGS 吸收层厚度较文献报 道稍薄, 较厚的 CIGS 吸收层组装的电池效率并不 高, 可能是由于制备的 CIGS 薄膜的晶粒尺寸较小, 影响了载流子的迁移距离, 较厚的吸收层反而影响 了载流子的收集效果。

\section{3 结论}

1) 对比了添加 CuSe 与否对 CIGS 靶材烧结工 艺的影响, 结果表明两种配方的原料粉均可以在适 当温度下烧结获得纯净物相的 CIGS 靶, 而原料中 CuSe 的加入可以显著降低体系的烧结温度。与不添 加 CuSe 的体系相比, 烧结温度降低了 $50^{\circ} \mathrm{C}$ 。

2) 采用 CIGS 靶材, 一步溅射并经热处理获得 的 CIGS 薄膜组装的电池，其转换效率达到 $9.6 \%$ 。

\section{参考文献:}

[1] SEIKE S, SHIOSAK K, KURAMOTO M, et al. Development of high-efficiency CIGS integrated submodules using in-line deposition technology. Solar Energy Material \& Solar Cells, 2011, 95(1): 254-256.

[2] ZHANG L, LIU F F, LI F Y, et al. Structural, optical and electrical properties of low-temperature deposition $\mathrm{Cu}\left(\mathrm{In}_{x} \mathrm{Ga}_{1-x}\right) \mathrm{Se}_{2}$ thin films. Solar Energy Material \& Solar Cells, 2012, 99(10): 356-361.

[3] MOON D G, AHN S J, YUN J H, et al. Ex-situ and in-situ analyses on reaction mechanism of $\mathrm{CuInSe}_{2}$ (CIS) formed by selenization of sputter deposited CuIn precursor with Se vapor. Solar Energy Material \& Solar Cells, 2011, 95(10): 2786-2794.

[4] LI Z Q, LIU Q Q, LI J J, et al. Growth of Zn doped Cu(In, Ga)Se thin films by RF sputtering for solar cell applications. Solid-State Electronics, 2012, 68: 80-84.

[5] OUYANG L Q, ZHUANG DM, ZHAO M, et al. Cu(In, Ga)Se ${ }_{2}$ solar cell with $16.7 \%$ active-area efficiency achieved by sputtering from a quaternary target. Physica Status Solidi A, 2015, 212(8): 1774-1778.
[6] SHUI J H, LI Z Q, ZHANG D W, et al. Fabrication of Cu(In, $\mathrm{Ga} \mathrm{Se}_{2}$ thin films by sputtering from a single quaternary chalcogenide target. Progress in Photovoltaics: Research and Applications, 2011, 19(2): 160-164.

[7] 梅 迪. 单靶磁控一步溅射制备 CIGS 薄膜. 成都: 电子科技大 学硕士学位论文, 2011.

[8] 孔 慧. 单靶磁控溅射制备 CIGS 太阳能电池材料的研究. 长春: 东北师范大学博士学位论文, 2014.

[9] SURYSNARAYANA C, IVANOV E, NOUFI R, et al. Synthesis and processing of a Cu-In-Ga-Se sputtering target. Thin Solid Films, 1998, 332: 340-344.

[10] 田村友哉, 高见英生, 生泽正克, 等. Cu-In-Ga-Se 四元合金溅 射靶，中国, C23C14/34, ZL201080048413.7, 2014.08.27。

[11] HSU W H, HSIANG H I, YEN F C, et al. Low-temperature sintered $\mathrm{CuIn}_{0.7} \mathrm{Ga}_{0.3} \mathrm{Se}_{2}$ prepared by colloidal processing. Journal European Ceramic Society, 2012, 32(14): 3753-3757.

[12] 王东生, 黄开盛, 龙飞, 等. 太阳能电池铜铟镓硒薄膜关键靶材及 其制备方法. 中国, C23C14/06, ZL200810073549.8. 2008.09.10.

[13] ZHANG N, ZHUANG D M, ZHANG G. An investigation on preparation of CIGS targets by sintering process. Material Science Engineering: B, 2010, 166: 34-40.

[14] LI XIAO-LONG, ZHUANG DA-MING, ZHAO MING, et al. Preparation and performance research of high dense rate of $\mathrm{Cu}(\mathrm{In}, \mathrm{Ga}) \mathrm{Se}_{2}$ ceramic target. Journal of Solar Energy, 2013, 32(12): 2196-2199.

[15] BEMARDini G P, CATANI A. The Cu-Se system. Mineralium Deposita, 1968, 3(4): 375-380.

[16] ROY S, GUHA P, KUNDU S N, et al. Characterization of Cu(In, $\mathrm{Ga} \mathrm{Se}_{2}$ films by Raman scattering. Materials Chemistry and Physics, 2002, 73(1): 24-30.

[17] 乔英杰. 材料合成与制备. 北京：国防工业出版社，2012: 160-178.

[18] KINGERY W D. Surface tension of some liquid oxides and their temperature coefficients. Journal of the American Ceramic Society, 1959, 42(1): 6-10.

[19] KINGERY W D, WOULBROUN J M, CHARVAT F R. Effects of applied pressure on densification during sintering in the presence of a liquid phase. Journal of the American Ceramic Society, 1963, 46(8): 391-395. 